\title{
Qualidade de vida em pacientes com retinopatia diabética proliferativa
}

\section{Quality of life in patients with proliferative diabetic retinopathy}

Regina Halfeld Furtado de Mendonça', Karina Franco Zihlmann², Mayara Limeira Freire ${ }^{3}$, Regina Carvalho de Salles Oliveira", Newton Kara José ${ }^{5}$

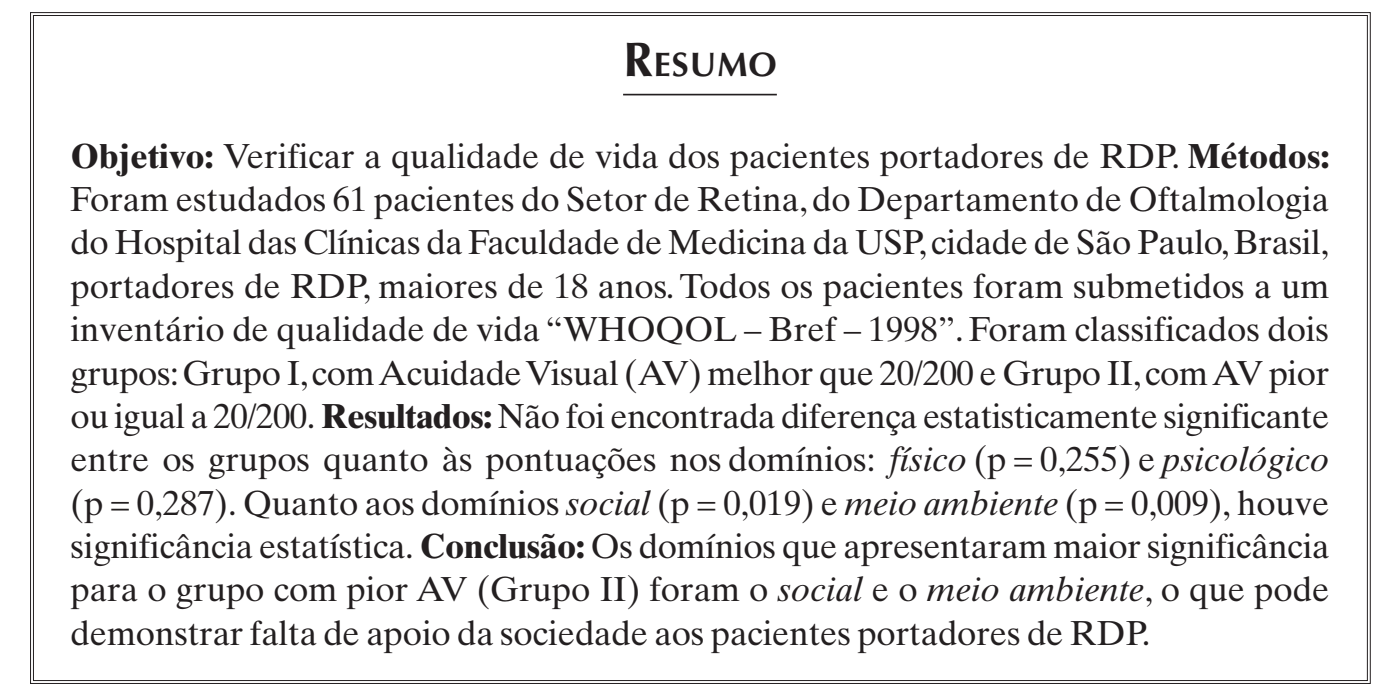

Descritores: Diabetes mellitus; Retinopatia diabética; Qualidade de vida

\footnotetext{
${ }^{1}$ Doutora, Médica assistente da Fundação Faculdade de Medicina da Universidade de São Paulo - USP - São Paulo (SP) - Brasil; Professora Convidada da Universidade La Sapienza, Roma - Italia;

${ }^{2}$ Doutoranda, Psicóloga da Divisão de Psicologia do Instituto Central do Hospital das Clínicas da Faculdade de Medicina da Universidade de São Paulo - USP - São Paulo (SP) - Brasil;

${ }^{3}$ Psicóloga, estagiária em Psicologia Hospitalar pelo Instituto Central do Hospital das Clínicas da Faculdade de Medicina da Universidade de São Paulo - USP - São Paulo (SP) - Brasil;

${ }_{5}^{4}$ Pedagoga, Doutora pela Faculdade de Medicina da Universidade de São Paulo - USP - São Paulo (SP) - Brasil;

${ }^{5}$ Professor Titular do Departamento de Oftalmologia da Faculdade de Medicina da Universidade de São Paulo - USP - São Paulo (SP)

- Brasil e da Faculdade de Ciências Médicas da UNICAMP - Campinas (SP) - Brasil.

Trabalho realizado no Departamento de Oftalmologia do Hospital das Clínicas da Faculdade de Medicina da Universidade de São Paulo - USP - São Paulo (SP), Brasil.

Recebido para publicação em: 3/4/2008 - Aceito para publicação em 16/7/2008
} 


\section{INTRODUÇãO}

$\mathbf{O}$ diabetes mellitus (DM) faz parte de um grupode doenças metabólicas caracterizadas porhiperglicemia resultante de distúrbios na secreção de insulina e/ou na ação da insulina. A hiperglicemia associa-se a complicações, disfunções e insuficiência de vários órgãos, como olhos, rins, nervos, cérebro, coração e vasos sangüíneos. As alterações metabólicas podem estar relacionadas a defeitos de secreção e/ou ação da insulina envolvendo processos patogênicos específicos, como a destruição das células b do pâncreas. Estima-se 35 milhões de pessoas com DM no mundo (Organização Panamericana de Saúde - OPS), sendo 19 milhões de pessoas na América Latina e Caribe. Essa doença de grande prevalência mundial mostra-se como um grave problema de Saúde Pública, caracterizando-se como um desafio para os sistemas de saúde, na medida em que, nos casos avançados, a DM pode resultar em complicações cardiovasculares, alterações visuais, amputação de membros inferiores e doenças renais ${ }^{(1)}$.

A retinopatia diabética $(\mathrm{RD})$ é uma complicação muito comum do diabetes. É uma grande causa de cegueira no mundo, decorrente da alteração dos vasos retinianos e é o principal fator de cegueira na faixa etária dos 20 aos 50 anos. A fisiopatologia da RD ainda não é totalmente esclarecida, mas acredita-se que a glicemia elevada piora o quadro clínico. A prevalência da RD aumenta com a duração da doença e com a idade do paciente. Após 20 anos de doença, perto de $99 \%$ dos portadores de DM insulino dependentes e $60 \%$ dos portadores de DM não insulino dependentes têm algum grau de $\mathrm{RD}^{(2)}$.

A RD consiste na manifestação retiniana de edema, exsudatos e hemorragias ${ }^{(3)}$. Pode ser classificada em retinopatia diabética não proliferativa (RDNP) e proliferativa (RDP). As hemorragias podem ser desencadeadas por proliferação neurovascular, a qual representa um fator importante na patogênese de complicações que podem levar à perda visual. Por esse motivo, pacientes com RDP devem ser submetidos a exames oftalmológicos freqüentes, com um adequado controle da glicemia, diagnóstico e tratamento precoce, para evitar o risco de perda visual ${ }^{(4)}$.

Atualmente, podemos encontrar vários estudos relacionando os efeitos do DM sobre a auto-estima do paciente, sobre sua capacidade de aderir ao tratamento e sobre sua qualidade de vida. Neste trabalho, enfocamos a questão da qualidade de vida em pacientes com RPDP.

A Qualidade de Vida pode ser diretamente relacionada com o conceito de auto-estima e com o bemestar pessoal, que englobam uma série de aspectos envolvendo a capacidade funcional, o nível socioeconômico, o estado emocional, a interação social, a atividade intelectual, o autocuidado, o suporte familiar, o próprio estado de saúde, os valores éticos e culturais, a religiosidade, o estilo de vida, a satisfação profissional e/ou as atividades diárias e $\mathrm{o}$ ambiente em que se vive. Trata-se de um conceito subjetivo e bastante ligado a esses aspectos citados, dependendo do contexto em que cada indivíduo está inserido, como nível sociocultural, faixa etária e suas aspirações pessoais ${ }^{(5)}$.

Com o crescente número de estudos na área, a Organização Mundial de Saúde, no ano de 1948, conceituou, de forma mais completa, a expressão, descrevendo Qualidade de Vida como a percepção do próprio indivíduo acerca de sua posição na vida, envolvendo contextos culturais e sistemas de valores em que está inserido, bem como seus objetivos, expectativas, padrões e preocupações pessoais. A partir da década de 1970, a avaliação da Qualidade de Vida (QV) cresceu de uma atividade relativamente restrita para uma disciplina formal, com uma estrutura teórica coesa, métodos consagrados e diversas aplicações. Nos últimos anos, a Qualidade de Vida tornou-se uma variável útil para determinar o impacto global das doenças e dos tratamentos médicos, a partir da perspectiva do paciente $^{(6)}$.

A mensuração da QV possibilita a comparação entre estudos de tratamento, evidenciando características importantes que podem auxiliar na compreensão da escolha terapêutica, pois permite a identificação dos aspectos da QV afetados pela terapia proposta ${ }^{(7)}$. Dessa forma, os dados podem fornecer maiores informações em relação à resposta de diferentes pacientes ou grupos de pacientes; subsidiar o prognóstico de resultados terapêuticos individuais, tendo em vista a relação de QV e prognóstico clínico; auxiliar na avaliação da eficiência de um procedimento, por fornecer dados subjetivos significativos.

Outro aspecto importante dos estudos da QV é o fato de que a compreensão do impacto subjetivo dos tratamentos "como melhoras sintomatológicas, efeitos colaterais ou tóxicos, alterações na dinâmica social e econômica e aspectos da vida diária ou psicológica dos pacientes", na perspectiva deles próprios é de extrema 
importância para atender às suas necessidades ${ }^{(7)}$.

Alguns trabalhos com pacientes portadores de DM mostraram que essa doença pode afetar diretamente a QV. Pode-se constatar, nesses estudos, o alto nível de depressão nos pacientes. Dessa forma, o DM foi relacionado como um fator de risco no desenvolvimento da depressão ${ }^{(8)}$. Em um estudo comparativo da avaliação de aspectos psiquiátricos, pode-se observar que a avaliação da QV dos pacientes diabéticos apresentou um alto índice no domínio psicológico comparado com pacientes esquizofrênicos ${ }^{(9)}$. Assim, constatase a importância da avaliação da QV para melhor conhecimento do estado mental do paciente, favorecendo estratégias mais eficientes para a adesão ao tratamento.

Entretanto, no que concerne à Retinopatia Diabética, poucos trabalhos relacionando os aspectos subjetivos dos pacientes foram publicados. Pesquisas sobre essa questão poderiam, portanto, promover novas estratégias em relação aos tratamentos, facilitando a promoção de saúde aos pacientes e permitindo a construção de fatores favorecedores da qualidade de vida. É o que se pretende apresentar no presente trabalho, que investigou a qualidade de vida dos pacientes portadores de retinopatia diabética proliferativa (RDP).

\section{Métodos}

Trata-se de um estudo quantitativo, transversal, com uma amostra de conveniência de 61 pacientes, maiores de 18 anos, portadores de Retinopatia Diabética Proliferativa, do Ambulatório de Oftalmologia do Hospital das Clínicas da Faculdade de Medicina da USP, cidade de São Paulo, Brasil. Os pacientes assinaram um Termo de Consentimento Livre e esclarecido, e a pesquisa foi aprovada pelo Comitê de Ética em Pesquisa do Hospital das Clínicas (CAPPesq) com o n ${ }^{\circ}$ 1057/05. Todos os preceitos da Resolução 196/96 do Conselho Nacional da Saúde foram seguidos.

Os pacientes responderam ao inventário de qualidade de vida "WHOQOL - ABREVIADO - versão em português, dos instrumentos de avaliação de qualidade de vida - 1998", após a consulta de rotina com o médico oftalmologista. $\mathrm{O}$ entrevistador perguntava ao médico qual a acuidade visual do paciente e, em uma sala reservada, aplicava o questionário.

A QV pode ser avaliada por vários inventários. A necessidade de instrumentos de rápida aplicação e com características psicométricas satisfatórias fez com que o Grupo de Qualidade de Vida da OMS desenvolvesse uma versão abreviada do WHOQOL-100, o WHOQOL-bref $f^{(10)}$.

WHOQOL-Brefé composto por quatro domínios: físico, psicológico, relações sociais e meio-ambiente. Os dados obtidos pelo inventário WHOQOL-Bref foram analisados e digitados em planilha do Microsoft Excel e analisados pelo Software SPSS para Windows versão 12.0. Todos os pacientes responderam a todas as 26 questões que compunham o questionário e, dessa forma, a amostra pôde ser estudada integralmente ${ }^{(10-13)}$.

As variáveis qualitativas foram representadas por freqüência absoluta (n) e relativa (\%) e as quantitativas, por média, desvio padrão (dp), mediana, valores mínimo e máximo. A distribuição normal das pontuações dos domínios foi testada pela Prova de Kolmogorov-Smirnov. Em nenhum dos testes, essa hipótese foi rejeitada. A comparação entre os grupos I e II foi feita pelo teste $t$ Student para amostras independentes. Adotou-se o nível de significância de 0,05 (a = $5 \%$ ). Níveis descritivos (p) inferiores a esse valor foram considerados significantes e representados.

\section{Resultados}

A amostra foi composta por 61 pacientes do setor de Retina do Departamento de Oftalmologia do Hospital das Clínicas da Faculdade de Medicina da Universidade de São Paulo, portadores de RDP, com idades variando entre 30 e 73 anos e média de 58 anos ( $\mathrm{dp}=8$ anos), sendo $52,5 \%$ de homens e $47,5 \%$ de mulheres.

A maior parte dos pacientes $(70,4 \%)$ tinha primeiro grau completo ou incompleto e $54,1 \%$ dos pacientes era casado ou vivia com companheiro. Quanto à percepção de sua própria saúde, apenas $18 \%$ a consideravam boa e $52,5 \%$, muito ruim ou fraca.

A Tabela 1 mostra as medidas descritivas das características dos pacientes que fizeram parte da amostra.

Dos 61 pacientes avaliados, 28 (45,9\%) tinham acuidade visual (AV) melhor que 20/200 e 33 (54,1\%) tinham AV pior ou igual a 20/200.

A amostra foi dividida em dois grupos de interesse, formados segundo a AV dos pacientes: grupo I, com acuidade visual melhor que 20/200 e grupo II, com AV pior ou igual a $20 / 200$. O grupo I teve $64,3 \%$ de mulheres e o grupo II, $66,7 \%$ de homens. A média de idade, nos dois grupos, foi semelhante: 57 anos no grupo 
Tabela 1

\section{Características da amostra} de 61 pacientes com RDP

\begin{tabular}{lr}
\hline Variáveis & N (\%) \\
\hline Sexo & \\
Masculino & $32(52,5)$ \\
Feminino & $29(47,5)$ \\
Idade (anos) & \\
Média \pm d.p. & $57,8 \pm 8,5$ \\
Mínimo - Máximo & $30-73$ \\
Nível educacional & \\
Analfabeto & $8(13,1)$ \\
I grau incompleto & $24(39,3)$ \\
I grau completo & $19(31,1)$ \\
II grau incompleto & $2(3,3)$ \\
II grau completo & $4(6,6)$ \\
III grau incompleto & $1(1,6)$ \\
III grau completo & $3(4,9)$ \\
Estado civil & \\
Solteiro (a) & $7(11,5)$ \\
Casado (a) & $31(50,8)$ \\
Vivendo como casado (a) & $2(3,3)$ \\
Separado (a) & $3(4,9)$ \\
Divorciado (a) & $8(13,1)$ \\
Viúvo (a) & $8(13,1)$ \\
Não informado & $2(3,3)$ \\
Percepção geral da saúde pessoal & \\
Muito ruim & $7(11,5)$ \\
Fraca & $25(41,0)$ \\
Nem ruim, nem boa & $18(29,5)$ \\
Boa & $11(18,0)$ \\
\hline
\end{tabular}

Tabela 2

Características da amostra classificados segundo a acuidade visual

\begin{tabular}{|c|c|c|}
\hline Variáveis & $\begin{array}{c}\text { Grupo I } \\
(\mathbf{n}=\mathbf{2 8})\end{array}$ & $\begin{array}{r}\text { Grupo II } \\
(\mathbf{n}=\mathbf{3 3})\end{array}$ \\
\hline \multicolumn{3}{|l|}{ Sexo - n (\%) } \\
\hline Masculino & $10(35,7)$ & $22(66,7)$ \\
\hline Feminino & $18(64,3)$ & $11(33,3)$ \\
\hline \multicolumn{3}{|l|}{ Idade (anos) } \\
\hline Média \pm d.p. & $57,3 \pm 6,5$ & $58,2 \pm 9,9$ \\
\hline Mínimo - Máximo & $45-73$ & $30-73$ \\
\hline \multicolumn{3}{|l|}{ Nível educacional - n (\%) } \\
\hline Analfabeto & $3(10,7)$ & $5(15,2)$ \\
\hline I grau incompleto & $12(42,9)$ & $12(36,4)$ \\
\hline I grau completo & $11(39,3)$ & $8(24,2)$ \\
\hline II grau incompleto & $1(3,6)$ & $1(3,0)$ \\
\hline II grau completo & $1(3,6)$ & $3(9,1)$ \\
\hline III grau incompleto & & $1(3,0)$ \\
\hline III grau completo & & $3(9,1)$ \\
\hline \multicolumn{3}{|l|}{ Estado civil - n (\%) } \\
\hline Solteiro (a) & $3(10,7)$ & $4(12,1)$ \\
\hline Casado (a) & $13(46,4)$ & $18(54,5)$ \\
\hline Vivendo como casado (a) & $1(3,6)$ & $1(3,0)$ \\
\hline Separado (a) & $2(7,1)$ & $1(3,0)$ \\
\hline Divorciado (a) & $5(17,9)$ & $3(9,1)$ \\
\hline Viúvo (a) & $4(14,3)$ & $4(12,1)$ \\
\hline Não informado & & $2(6,1)$ \\
\hline \multicolumn{3}{|l|}{ Saúde - n (\%) } \\
\hline Muito ruim & $2(7,1)$ & $5(15,2)$ \\
\hline Fraca & $12(42,9)$ & $13(39,4)$ \\
\hline Nem ruim, nem boa & $8(28,6)$ & $10(30,3)$ \\
\hline Boa & $6(21,4)$ & $5(15,2)$ \\
\hline
\end{tabular}

Tabela 3 Medidas descritivas das pontuações nos diferentes domínios
da escala WHOQOL-BREF na amostra de 61 pacientes

\begin{tabular}{lrrrrr}
\hline Domínios $(\mathbf{n}=61)$ & \multicolumn{6}{l}{ WHOQOL - BREF } & & & \\
\cline { 2 - 6 } & \multicolumn{1}{l}{ Média } & dp & Mediana & Mínimo & Máximo \\
\hline Físico & 47,42 & 17,52 & 46,43 & 10,71 & 82,14 \\
Psicológico & 53,62 & 19,61 & 58,33 & 0 & 83,33 \\
Social & 53,55 & 20,32 & 58,33 & 0 & 100,00 \\
Meio ambiente & 45,80 & 13,95 & 46,88 & 6,25 & 78,13 \\
\hline
\end{tabular}

I e 58 anos no grupo II. Como na amostra total a maior parte dos pacientes $(82,2 \%$ e $60,6 \%$ respectivamente nos grupos I e II) tinha primeiro grau completo ou incompleto. Entre $50 \%$ e $60 \%$ dos pacientes era casado ou vivia com companheiro.

Quanto à percepção de sua própria saúde, 21,4\% no grupo I e $15,2 \%$ no grupo II, a consideravam boa e $50,0 \%$ e $54,6 \%$ respectivamente muito ruim ou fraca.

Na Tabela 2, se encontram as medidas descritivas das características dos pacientes, divididos segundo a AV.

$\mathrm{Na}$ tabela 3, se encontram as medidas descriti- 
Tabela 4

Medidas descritivas das pontuações nos diferentes domínios da escala WHOQOL-BREF na amostra de 61 pacientes divididos em dois grupos

\begin{tabular}{llll}
\hline $\begin{array}{l}\text { Escala WHOQOL-BREF } \\
\text { Domínios }\end{array}$ & Grupo I(n = 28) & Grupo II(n= 33) & Comparação \\
\hline Físico & & & \\
$\quad$ Média \pm d.p. & $50,51 \pm 17,73$ & $45,35 \pm 17,26$ & $\mathrm{p}=0,255$ \\
Mediana & 50,00 & 46,43 & \\
Mínimo - Máximo & $10,71-82,14$ & $14,29-82,14$ & \\
Psicológico & & & \\
$\quad$ Média \pm d.p. & $56,55 \pm 21,71$ & $51,14 \pm 17,59$ & $\mathrm{p}=0,287$ \\
Mediana & 64,58 & 54,17 & \\
Mínimo - Máximo & $0-83,33$ & $16,67-83,33$ & \\
Social & & & \\
Média \pm d.p. & $60,12 \pm 20,20$ & $47,98 \pm 18,98$ & $\mathrm{p}=0,019 *$ \\
Mediana & 58,33 & 50,00 & \\
Mínimo - Máximo & $0-100$ & $16,67-75,00$ & $\mathrm{p}=0,009 *$ \\
Meio ambiente & & & \\
Média \pm d.p. & $50,78 \pm 14,09$ & $41,57 \pm 12,54$ & \\
Mediana & 51,56 & 40,63 & \\
Mínimo - Máximo & $25,00-78,13$ & $6,25-65,63$ & \\
* Nível de signifiĉncia de 0,05 & & &
\end{tabular}

vas das pontuações dos domínios da escala WHOQOL - BREF aplicada na amostra de 61 pacientes.

$\mathrm{Na}$ Tabela 4, se encontram as medidas descritivas das pontuações dos domínios da escala WHOQOL - BREF aplicada na amostra de pacientes, divididos segundo a AV (grupos I e II).

Não foi encontrada diferença estatisticamente significante entre os grupos quanto às pontuações nos domínios: físico $(\mathrm{p}=0,255)$ e psicológico $(\mathrm{p}=0,287)$. Foi encontrada diferença estatisticamente significante entre os grupos nos domínios social $(\mathrm{p}=0,019)$ e meio-ambiente $(\mathrm{p}=0,009)$. Em ambos os domínios, as médias de pontuação do grupo I foram significantemente maiores do que as médias do grupo II.

\section{Dıscussão}

Considerando-se que o conceito de Qualidade de Vida envolve contextos sócioculturais e sistemas de valores, pode-se inferir que, na situação de doença incapacitante como a RDP, seria esperado que o grupo II (pacientes com pior AV) apresentasse piores escores em todos os domínios (físico,psicológico, social e meio ambiente), especialmente nos domínios físico (no campo da sensação de bem-estar físico e capacidade de lo- comoção e trabalho) e no domínio psicológico (no campo da auto-percepção e auto-estima dos indivíduos), quando comparado ao grupo I (pacientes com melhor AV). Surpreendentemente, não constatamos diferenças estatisticamente significantes entre os grupos, pelo menos no que concerne ao domínio físico e psicológico.

Observou-se, entretanto, que a qualidade de vida da população estudada sofre maior impacto na relação dos sujeitos com o mundo (domínio das relações sociais) e com o ambiente físico (domínio meio ambiente). Os resultados apontaram diferença estatisticamente significante entre os grupos I e II, nos domínios social $(\mathrm{p}=0,019)$ e meio-ambiente $(\mathrm{p}=0,009)$.

Vale salientar que as ações educativas para o controle da DM junto ao paciente, família e comunidade são de extrema importância, tendo em vista que um dos maiores problemas no controle da doença é a dificuldade de acesso ao Centro de Saúde ${ }^{(14)}$. Nosso trabalho também mostra que há maior impacto no domínio social e no de meio ambiente, especialmente nos pacientes considerados "mais graves" (com pior AV). Dessa forma, pode-se enfatizar a importância da assistência à saúde, na questão da prevenção, orientação e acessibilidade aos serviços de saúde, principalmente em relação a esses pacientes com RPD, portadores de necessidades especiais. 
Talvez seja possível compreender que, para eles, a questão da acessibilidade pode representar uma questão de grande impacto na QV. Sobre a acessibilidade pode-se dizer que esta significa o grau de facilidade com que as pessoas obtêm os cuidados de saúde, e representa o centro do funcionamento dos serviços de saúde, no que concerne ao atendimento direto a consultas médicas e hospitalização, como também ao indireto, por meio da realização de exames preventivos e diagnósticos. A acessibilidade ultrapassa as condições e distribuições geográficas de acesso ao paciente, porque também compreende questões como o acolhimento por parte dos profissionais, disponibilidade dos serviços às necessidades dos pacientes, entre outras questões que visam à promoção da saúde dos que procuram esses serviços ${ }^{(15)}$.

Dessa forma esse tipo de demanda, mais específica, precisaria ter "voz", principalmente em um país em desenvolvimento como o Brasil, com grandes carências de infra-estrutura de serviços públicos de qualidade. A maioria (52,5\%) dos pacientes entrevistados afirmou que seu estado de saúde geral era muito ruim ou fraco. Trata-se, portanto, do investimento e cuidado nas áreas onde há, de fato, uma demanda, para minimizar o sofrimento e desconforto causado pela doença $^{(1)}$.

Esta pesquisa tem o mérito de contribuir para a investigação da Retinopatia Diabética Proliferativa e avaliação da Qualidade de Vida em pacientes com DM, uma vez que se observa a escassez de trabalhos na literatura nacional. Embora, a amostra seja relativamente pequena, ainda assim, foi possível estabelecer diferenças estatisticamente relevantes, o que pode indicar que esses fatores observados têm, de fato, grande relevância para o tema. O instrumento de mensuração de qualidade de vida da OMS pode mostrar-se útil para ampliar a visão da referida temática, bem como impulsionar intervenções que possibilitem uma melhora na QV desses pacientes.

É interessante notar que as grandes diferenças "práticas" entre pacientes com distinta AV não foram observadas na auto-avaliação da qualidade de vida, pelo menos, quanto aos domínios físico e psicológico. A diferença fundamental entre os grupos está relacionada com suas demandas na obtenção de recursos sociais e ambientais para suprir suas necessidades. Tal diferença, presente em um país repleto de grandes diferenças sócioeconômicas, como é o caso do Brasil, pode nos levar a uma reflexão crítica sobre o papel do poder pú- blico e sua responsabilidade, em especial a portadores de DM, que representa um dos principais problemas de saúde mundial.

\section{Abstract}

Objective: To verify the Quality of life for the Proliferative Diabetic Retinopathy (PDR) patients. These subjects have a higher risk for severe visual loss, due to the vascular retina complications. Methods: We evaluated the Quality of Life of 61 PDR patients, over 18 years old, who have been followed up in the Ophthalmology Clinic of the Clinical Hospital of Medicine School of São Paulo, Brazil. All patients data were obtained using a questionnaire of health quality of life "WHOQOL-Bref-Portuguese version - 1998", and the statistical analysis was done using SPSS program. The 61 patients were classified in two groups: Group I, with 28 patients (46\%), with better visual acuity (VA) of 20/200 and Group II, with 33 patients (54\%), with worse or equal VA of 20/200. Results: No statistical significant difference (SSD) was observed between the groups, regarding to physical domain ( $p=$ $0,255)$ and psychological domain $(p=0,287) . S S D$ was observed between the groups, regarding to social domain $(p=0.019)$ and environment domain ( $p=0.009)$. Conclusion: Patients with poor VA can be equivalent with those with better vision in physical and psychological domains (no SSD). The SSD regarding to social and environment domains demonstrate the lack of social support for people living with severe visual loss.

Keywords: Diabetes mellitus; Diabetic retinopathy; Quality of life

\section{REFERÊNCIAS}

1. Aguilar PIDSG. Qualidade de vida em pessoas com diabetes Mellitus tipo 2 [tese doutorado]. Ribeirão Preto: Escola de Enfermagem de Ribeirão Preto da Universidade de São Paulo; 2004.

2. Moreira Jr CA, Ávila M. Retinopatia diabética. In: Moreira Jr CA, Ávila M. Retina e vítreo. Rio de Janeiro: Cultura Médica; 2000.

3. Corrêa ZMS, Eagle Júnior R. Aspectos patológicos da retinopatia diabética. Arq Bras Oftalmol. 2005; 68(3): 410-4.

4. Elfervig LS, Elfervig JL. Proliferative diabetic retinopathy. Insight. 2001; 26(3): 88-91; quiz 92-3. Review.

5. Vecchia RD, Ruiz T, Bocchi SCM, Corrente JE. Qualidade de vida na terceira idade: um conceito subjetivo. Rev Bras Epidemiol. 2005; 8(3):246-52. 
6. Berlim MT, Fleck MP. "Quality of life": a brand new concept for research and practice in psychiatry. Rev Bras Psiquiatr. 2003; 25(4):249-52.

7. Blay SL, Merlin MS. Desenho e metodologia de pesquisa em qualidade de vida. In: Diniz DP, Schor N, organizadores. Qualidade de vida: São Paulo: Manole, 2006. p. 19-30. v. 1. [Guias de Medicina Ambulatorial e Hospitalar].

8. Eren I, Erdi O, Sahin M. The effect of depression on quality of life of patients with type II diabetes mellitus. Depress Anxiety. 2008; 25(2):98-106.

9. Akvardar Y, Akdede BB, Ozerdem A, Eser E, Topkaya S, Alptekin K. Assessment of quality of life with the WHOQOLBREF in a group of Turkish psychiatric patients compared with diabetic and healthy subjects. Psychiatry Clin Neurosci. 2006; 60(6): 693-9.

10. Fleck MPA, Louzada S, Xavier M, Chachamovich E, Vieira G, Santos L, Pinzon V. Aplicação da versão em português do instrumento abreviado de avaliação da qualidade de vida "WHOQOL-bref". Rev Saude Publica = J Public Health. 2000; 34(2):178-83.

11. Fleck MPA, Leal OF, Louzada S, Xavier M, Chachamovich E, Vieira G, Santos L, Pizon V. Desenvolvimento da versão em português do instrumento de avaliação de qualidade de vida da OMS (WHOQOL-100). Rev Bras Psiquiatr. 1999; 21(1):19-28.
12. Fleck MPA, Louzada S, Xavier M, Chachamovich E, Vieira G, Santos L, Pinzon V. Aplicação da versão em português do instrumento de avaliação de qualidade de vida da Organização Mundial da Saúde (WHOQOL-100). Rev Saude Publica = J Public Health. 1999; 33(2):198-205.

13. Fleck MPA, Lima AFBS, Louzada S, Schestasky G, Henriques A, Borges VR, Camey S. Associação entre sintomas depressivos e funcionamento social em cuidados primários à saúde. Rev Saude Publica $=$ J Public Health. 2002; 36(4):431-8.

14. Faeda A, Leon CGRMP. Assistência de enfermagem a um paciente portador de Diabetes Mellitus. Rev Bras Enferm. 2006; 59(6): 818-21.

15. Travassos C, Martins, M. Uma revisão sobre os conceitos de acesso e utilização de servidos de saúde. Cad Saude Publica = Rep Public Health. 2004; 20(Supl 2):190-8.

\section{ENDEREÇO PARA CORRESPONDÊNCIA: \\ Regina Halfeld Furtado de Mendonça \\ Via Muzio Attendolo, 31 \\ 00176 - Roma - Itália \\ halfeld@libero.it}

